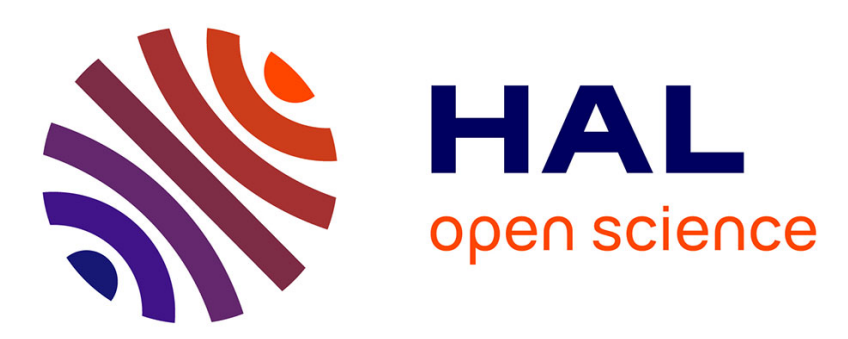

\title{
Towards a Framework for Algorithm Recognition in Binary Code
}

\author{
Frédéric Mesnard, Etienne Payet, Wim Vanhoof
}

\section{To cite this version:}

Frédéric Mesnard, Etienne Payet, Wim Vanhoof. Towards a Framework for Algorithm Recognition in Binary Code. 18th International Symposium on Principles and Practice of Declarative Programming (PPDP), Sep 2016, Edinburgh, United Kingdom. pp.202-213. hal-01451686

\section{HAL Id: hal-01451686 \\ https://hal.univ-reunion.fr/hal-01451686}

Submitted on 5 Nov 2018

HAL is a multi-disciplinary open access archive for the deposit and dissemination of scientific research documents, whether they are published or not. The documents may come from teaching and research institutions in France or abroad, or from public or private research centers.
L'archive ouverte pluridisciplinaire $\mathbf{H A L}$, est destinée au dépôt et à la diffusion de documents scientifiques de niveau recherche, publiés ou non, émanant des établissements d'enseignement et de recherche français ou étrangers, des laboratoires publics ou privés. 


\section{Towards a Framework for Algorithm Recognition in Binary Code}

\author{
Frédéric Mesnard \\ LIM, université de la Réunion, France \\ frederic.mesnard@univ-reunion.fr
}

\author{
Étienne Payet \\ LIM, université de la Réunion, France \\ etienne.payet@univ-reunion.fr
}

\author{
Wim Vanhoof \\ Faculté d'informatique, université de \\ Namur, Belgium \\ wim.vanhoof@unamur.be
}

\begin{abstract}
Algorithm recognition, which is the problem of verifying whether a program implements a given algorithm, is an important topic in program analysis. We propose an approach for algorithm recognition in binary code. For this paper, we have chosen the Dalvik Virtual Machine (DVM) bytecode. Given an algorithm $A$ that is compiled into a DVM method $M_{0}$, and a DVM program $P$ that includes a series of methods $\left\{M_{1}, \ldots, M_{n}\right\}$, the approach is able to identify those blocks $M_{i}$ from $P$ that essentially implement the algorithm $A$. The technique we propose first translates binary code into Horn clauses. Then we consider programs as implementing the same algorithm if their Horn clause representations can be reduced to a single common set of Horn clauses by means of a sequence of transformations.
\end{abstract}

Categories and Subject Descriptors D.2.4 [Software/Program Verification]: Formal methods.

Keywords Algorithm recognition.

\section{Introduction}

Algorithm recognition - which we could intuitively define as verifying whether a program implements a given algorithm (Wills 1993 ) - is an important topic in program analysis. Applications are diverse and range from program comprehension (Storey 2005) over plagiarism detection (Zhang et al. 2012) and malware detection (Zhang et al. 2014) to advanced analyses and optimisations such as the automatic detection of the best parallelisation strategy for a given code fragment (Martino and Iannello 1996).

How to identify whether a given piece of code implements a particular algorithm is a challenging question that can be studied from different angles. While it can generally be assumed that the algorithm to be recognised is known in the form of source code, whether this is the case for the codebase in which the search has to take place depends on the desired application. Consider for example a system for assessing whether students in a programming course have correctly implemented a particular sorting algorithm (Taherkhani and Malmi 2013); in that case one can safely assume the student's source code to be available and one can resort to techniques for assessing the similarity between two algorithms represented in source code. However, as an alternative example, consider a company that wishes to verify whether a competitor's software program uses a (proprietary) algorithm. In this case, the competitor's software might be written in a different programming language and is presumably only available as binary code. Consequently, the verification must be performed between some model of the algorithm and that of the binary code under scrutiny (Zhang et al. 2012).

In this work, we propose an approach for algorithm recognition in binary code, namely Google's Dalvik Virtual Machine (DVM) bytecode, making the approach directly applicable to (compiled) Android programs. A direct application of our approach resides in algorithm plagiarism detection, a topic that has - in contrast with so-called software plagiarism - received relatively little attention (Zhang et al. 2012). While software plagiarism is mainly about unlawfully reusing existing source code or libraries, algorithm plagiarism is more about unlawfully copying the ideas behind how a certain computation is done. Let us consider computing the maximum of three integer values as an almost ridiculously simple example. Figure 1 shows two different Java source methods that perform this computation. While the code of the two methods is quite different (and will as such presumably not be recognised as software plagiarism or software clones), the algorithm that is implemented by both methods is essentially the same. Indeed, in both cases one compares $z$ with the maximum of $x$ and $y$. In the middle method code this is more explicit by the presence of the auxiliary variable, but the left-hand side method does essentially the same. While the example is, admittedly, too simple to speak of algorithm plagiarism, for several other applications (for example in the context of program understanding or program refactoring) it might nevertheless be desirable to recognize that the algorithm underlying both methods is the same.

While the notion of two algorithms being the same is not easily defined, and the mere existence of equivalence classes of programs implementing the same algorithm is even subject to debate (Blass et al. 2009), a pragmatic approach that is often taken in algorithm recognition (Metzger and Wen 2000) is to consider programs as implementing the same algorithm if they can be reduced to one another by means of a sequence of syntactical transformations. While our approach is based on program transformation and targets algorithm recognition in binary code, one of its cornerstones is the fact that we use Horn clauses to represent a model of the algorithm as well as of the compiled code under scrutiny. This has several advantages:

- Horn clauses are a suitable abstraction that is in between binary code and a more high-level programming language. This is in line with recent work (Gange et al. 2015) in which the use of Horn Clauses as a universal intermediate language has been advocated. In that work the general idea is to compile a program (written in an arbitrary language) first into Horn clauses, then 
$p_{0}\left(V_{0}, V_{1}, V_{2}, V_{3}, V_{4},\langle A, I\rangle,\left\langle A^{\prime}, I\right\rangle\right) \leftarrow$

$\left\{V_{2} \geq V_{4}, V_{2} \geq V_{3}, A^{\prime}=A\left\{0 \leftarrow V_{2}\right\}\right\}$

$p_{0}\left(V_{0}, V_{1}, V_{2}, V_{3}, V_{4},\langle A, I\rangle,\left\langle A^{\prime}, I\right\rangle\right) \leftarrow$

$\left\{V_{2}<V_{3}, V_{3} \geq V_{4}, A^{\prime}=A\left\{0 \leftarrow V_{3}\right\}\right\}$.

$p_{0}\left(V_{0}, V_{1}, V_{2}, V_{2}, V_{4},\langle A, I\rangle,\left\langle A^{\prime}, I\right\rangle\right) \leftarrow$

$\left\{V_{2}<V_{4}, A^{\prime}=A\left\{0 \leftarrow V_{4}\right\}\right\}$.

$p_{0}\left(V_{0}, V_{1}, V_{2}, V_{3}, V_{4},\langle A, I\rangle,\left\langle A^{\prime}, I\right\rangle\right) \leftarrow$

$\left\{V_{2}<V_{4}, V_{3}<V_{2}, A^{\prime}=A\left\{0 \leftarrow V_{4}\right\}\right\}$.

$p_{0}\left(V_{0}, V_{1}, V_{2}, V_{3}, V_{4},\langle A, I\rangle,\left\langle A^{\prime}, I\right\rangle\right) \leftarrow$

$\left\{V_{2}<V_{3}, V_{3}<V_{4}, A^{\prime}=A\left\{0 \leftarrow V_{4}\right\}\right\}$

$$
\begin{array}{r}
p_{1}\left(V_{0}, V_{1}, V_{2}, V_{3}, V_{4}, V_{5},\langle A, I\rangle,\left\langle A^{\prime}, I\right\rangle\right) \leftarrow \\
\left\{V_{3} \geq V_{4}, V_{3} \geq V_{5}, A^{\prime}=A\left\{0 \leftarrow V_{3}\right\}\right\} . \\
p_{1}\left(V_{0}, V_{1}, V_{2}, V_{3}, V_{4}, V_{5},\langle A, I\rangle,\left\langle A^{\prime}, I\right\rangle\right) \leftarrow \\
\left\{V_{4} \geq V_{5}, V_{3}<V_{4}, A^{\prime}=A\left\{0 \leftarrow V_{4}\right\}\right\} . \\
p_{1}\left(V_{0}, V_{1}, V_{2}, V_{3}, V_{4}, V_{5},\langle A, I\rangle,\left\langle A^{\prime}, I\right\rangle\right) \leftarrow \\
\left\{V_{3} \geq V_{4}, V_{3}<V_{5}, A^{\prime}=A\left\{0 \leftarrow V_{5}\right\}\right\} . \\
p_{1}\left(V_{0}, V_{1}, V_{2}, V_{3}, V_{4}, V_{5},\langle A, I\rangle,\left\langle A^{\prime}, I\right\rangle\right) \leftarrow \\
\left\{V_{4}<V_{5}, V_{3}<V_{4}, A^{\prime}=A\left\{0 \leftarrow V_{5}\right\}\right\} .
\end{array}
$$

Figure 3. Horn clause representation of the methods of Figure 1, obtained by decompilation.

clauses. Section 3 provides the formal framework enabling the algorithm recognition process as described, and the formal definition of what it means, within this framework, when two program fragments implement the same algorithm. Section 4 summarizes related work and Section 5 concludes with a discussion of future work.

\section{From Binary Code to Clauses}

Android programs are written in Java. They are compiled to the Google's Dalvik Virtual Machine (DVM) bytecode format before installation on a device. We assume the reader familiar with the basic concepts of object-oriented programming and the Android platform (Android developers). Here, we briefly describe the DVM, see (ART and Dalvik) for a complete presentation.

Unlike the Java Virtual Machine (Lindholm and Yellin 1999) which is stack-based, the DVM is register-based. It runs a Dalvik bytecode program by keeping an activation stack of frames. Each frame is created by a method call, survives until the end of the call and uses its own registers. An invoked method cannot affect the registers in the frame of the invoking method. Any call to a same method always produces a frame with a same number of registers. For each method $\omega$, this number is statically known. We denote it as reg $(\omega)$ and we refer to it as the number of registers used by $\omega$.

The memory of the system contains objects, connected through pointers. To simplify the presentation, we do not consider array nor interface types and only allow integers as values of basic types.

Definition 1. The set of values is $\mathbb{Z} \cup \mathbb{L}$, where $\mathbb{Z}$ is the set of integers and $\mathbb{L}$ is the set of memory locations. A frame of the DVM is a pair $\langle v, \mu\rangle$ where $v$ is a sequence of values, called registers, numbered from 0 upwards and $\mu$ is a memory, or heap, that maps locations into objects. An object is a pair $\langle\kappa, f\rangle$ where $\kappa$ is a class identifier i.e., an index (integer) in the class definition list of the program, and $f$ is a sequence of values, called fields, numbered from 0 upwards; we say that it belongs to class $\kappa$ or that it is an instance of class $\kappa$ or has class $\kappa$. We let o. $\kappa$ and o.f respectively denote the class and fields of an object $o$. We require that there are no dangling pointers i.e., $v \cap \mathbb{L} \subseteq \operatorname{dom}(\mu)$ and $\mu(\ell) . f \cap \mathbb{L} \subseteq \operatorname{dom}(\mu)$ for every $\ell \in \operatorname{dom}(\mu)$. The set of all classes is denoted by $\mathbb{K}$ and it is partially ordered by the subclass relation (we consider that a class is a subclass of itself).

The Dalvik bytecode is strongly typed. Each value has a type and registers are statically typed.

Definition 2. The set of types of our simplified DVM is $\mathbb{T}=$ $\mathbb{K} \cup\{$ int, void $\}$. The void type can only be used as the return type of methods. A method signature is denoted by $\kappa \cdot m\left(t_{1}, \ldots, t_{p}\right) t$ standing for a method named $m$, defined in class $\kappa$, expecting $p$ explicit parameters of type, respectively, $t_{1}, \ldots, t_{p}$ and returning a value of type $t$, or returning no value when $t=$ void.

A non-static method $\kappa \cdot m\left(t_{1}, \ldots, t_{p}\right) t$ also has an implicit parameter of type $\kappa$ called this in the code of the method. So the actual number of parameters is $p+1$. We do not distinguish be- tween methods and constructors. A constructor is just a method named <init> and returning void. By a void method (resp. nonvoid method) we mean a method whose return type is void (resp. is not void). We do not consider static fields and methods. The extension of our definitions to them is not difficult.

Dalvik bytecode instructions work over frames and their execution affects the registers or the memory in the frames. Many are similar or only differ in the type or size of their operands. So we concentrate on a restricted set which exemplifies the operations that the DVM performs.

- const $d, c$ Writes constant $c$ into register $d$.

- move $d, s$ Writes the value of register $s$ into register $d$.

- add $d, s, c$ Writes the sum of the value of register $s$ and constant $c$ into register $d$.

- if-lt $i, j, q$ If the value of register $i$ is less than the value of register $j$ then jumps to program point $q$, otherwise executes the immediately following instruction.

- goto $q$ Jumps to program point $q$.

- invoke $S, \kappa \cdot m\left(t_{1}, \ldots, t_{p}\right) t$ where $S=s_{0}, s_{1}, \ldots, s_{p}$ is a sequence of register indexes. The value $v_{s_{0}}$ of register $s_{0}$, $\ldots, v_{s_{p}}$ of register $s_{p}$ are the actual parameters of the call. Value $v_{s_{0}}$ is called receiver of the call and must be 0 (the equivalent of null in Java) or the memory location of an object $o$. In the former case, the computation stops with an exception. Otherwise, a lookup procedure is started from the class of $o$ upwards along the superclass chain, looking for a method called $m$ expecting $p$ formal parameters of type $t_{1}, \ldots, t_{p}$, respectively, and returning a value of type $t$. It is guaranteed that such a method is found in a subclass of $\kappa$. That method is run from a new frame where the last $p+1$ registers are bound to $v_{s_{0}}, v_{s_{1}}, \ldots, v_{s_{p}}$, respectively, and the other ones to 0 .

- return Returns from a void method.

- return $s$ Returns from a non-void method with the value of register $s$ as result.

- move-result $d$ Writes the result of the most recent called method into register $d$. This instruction must immediately follow an invoke instruction.

- new-instance $d, \kappa$ Writes the memory location of a new, properly initialised, object of class $\kappa$ into register $d$.

- iget $d, i, j$ (resp. iput $s, i, j$ ) The value $v_{i}$ of register $i$ must be 0 or the memory location of an object $o$. If $v_{i}$ is 0 , the computation stops with an exception. Otherwise, the value of field $j$ of $o$ is written into register $d$ (resp. the value of register $s$ is written into the field $j$ of $o$ ).

We suppose that the Dalvik program $P$ under consideration consists of these instructions and that it is well-formed. For instance, each move-result immediately follows an instruction of the form 


$$
\begin{aligned}
q: \text { const } d, c & \mapsto\left\{p_{q}\left(\tilde{V}, M, M^{\prime}\right) \leftarrow\left\{V_{d}^{\prime}=c\right\} \cup i d_{-d}, p_{q+1}\left(\tilde{V}^{\prime}, M, M^{\prime}\right)\right\} \\
q: \text { move } d, s & \mapsto\left\{p_{q}\left(\tilde{V}, M, M^{\prime}\right) \leftarrow\left\{V_{d}^{\prime}=V_{s}\right\} \cup i d_{-d}, p_{q+1}\left(\tilde{V}^{\prime}, M, M^{\prime}\right)\right\} \\
q: \text { add } d, s, c & \mapsto\left\{p_{q}\left(\tilde{V}, M, M^{\prime}\right) \leftarrow\left\{V_{d}^{\prime}=V_{s}+c\right\} \cup i d_{-d}, p_{q+1}\left(\tilde{V}^{\prime}, M, M^{\prime}\right)\right\} \\
q: \text { goto } q^{\prime} & \mapsto\left\{p_{q}\left(\tilde{V}, M, M^{\prime}\right) \leftarrow i d, p_{q^{\prime}}\left(\tilde{V}^{\prime}, M, M^{\prime}\right)\right\} \\
q: \text { if-lt } i, j, q^{\prime} & \mapsto\left\{\begin{array}{l}
p_{q}\left(\tilde{V}, M, M^{\prime}\right) \leftarrow\left\{V_{i}<V_{j}\right\} \cup i d, p_{q^{\prime}}\left(\tilde{V}^{\prime}, M, \tilde{I}^{\prime}\right) \\
p_{q}\left(\tilde{V}, M, M^{\prime}\right) \leftarrow\left\{V_{i} \geq V_{j}\right\} \cup i d, p_{q+1}\left(\tilde{V}^{\prime}, M, M^{\prime}\right)
\end{array}\right\}
\end{aligned}
$$

Figure 4. Compilation of simple instructions.

$$
\begin{aligned}
& q: \text { ins } \mapsto\left\{\begin{array}{l|l}
\text { ins }=\text { invoke } s_{0}, \ldots, s_{p}, \kappa \cdot m\left(t_{1}, \ldots, t_{p}\right) t \\
p_{q}\left(\tilde{V},\langle A, I\rangle, M^{\prime}\right) \leftarrow\left\{\begin{array}{ll}
\left.V_{s_{0}}>0, \kappa^{\prime}=A\left[V_{s_{0}}, 0\right]\right\} \cup i d, & \kappa^{\prime} \text { is a subclass of } \kappa \\
p_{q_{\omega^{\prime}}}\left(\tilde{W},\langle A, I\rangle, M_{1}\right), & \omega^{\prime}=\operatorname{lookup}\left(\kappa \cdot m\left(t_{1}, \ldots, t_{p}\right) t, \kappa^{\prime}\right) \\
p_{q+1}\left(\tilde{V}^{\prime}, M_{1}, M^{\prime}\right) & \tilde{W}=0, \ldots, 0, V_{s_{0}}, \ldots, V_{s_{p}} \text { with }|\tilde{W}|=\operatorname{reg}\left(\omega^{\prime}\right)
\end{array}\right\}
\end{array}\right. \\
& q: \text { move-result } d \mapsto\left\{p_{q}\left(\tilde{V},\langle A, I\rangle, M^{\prime}\right) \leftarrow\left\{V_{d}^{\prime}=A[0]\right\} \cup i d_{-d}, p_{q+1}\left(\tilde{V}^{\prime},\langle A, I\rangle, M^{\prime}\right)\right\} \\
& q \text { :return } s \mapsto\left\{p_{q}\left(\tilde{V},\langle A, I\rangle,\left\langle A^{\prime}, I^{\prime}\right\rangle\right) \leftarrow\left\{A^{\prime}=A\left\{0 \leftarrow V_{s}\right\}, I^{\prime}=I\right\}\right\} \\
& q: \text { return } \mapsto\left\{p_{q}\left(\tilde{V},\langle A, I\rangle,\left\langle A^{\prime}, I^{\prime}\right\rangle\right) \leftarrow\left\{A^{\prime}=A, I^{\prime}=I\right\}\right\}
\end{aligned}
$$

Figure 5. Compilation of instructions related to method calls.

$$
\begin{gathered}
\text { ins }=\text { new-instance } d, \kappa \text { and } \text { objects of class } \kappa \text { have } n \text { fields } \\
q: \text { ins } \mapsto\left\{\begin{array}{r}
p_{q}\left(\tilde{V},\langle A, I\rangle, M^{\prime}\right) \leftarrow\left\{O[0]=\kappa, O[1]=0, \ldots, O[n]=0, A_{1}=A\{I \leftarrow O\},\right. \\
\left.V_{d}^{\prime}=I, I_{1}=I+1\right\} \cup i d_{-d}, p_{q+1}\left(\tilde{V}^{\prime},\left\langle A_{1}, I_{1}\right\rangle, M^{\prime}\right)
\end{array}\right\} \\
q: \text { iget } d, i, j \mapsto\left\{p_{q}\left(\tilde{V},\langle A, I\rangle, M^{\prime}\right) \leftarrow\left\{V_{i}>0, V_{d}^{\prime}=A\left[V_{i}, j+1\right]\right\} \cup i d_{-d}, p_{q+1}\left(\tilde{V}^{\prime},\langle A, I\rangle, M^{\prime}\right)\right\} \\
q: \text { iput } s, i, j \mapsto\left\{\begin{array}{r}
p_{q}\left(\tilde{V},\langle A, I\rangle, M^{\prime}\right) \leftarrow\left\{\begin{array}{r}
V_{i}>0, O=A\left[V_{i}\right], O_{1}=O\left\{j+1 \leftarrow V_{s}\right\}, \\
\left.A_{1}=A\left\{V_{i} \leftarrow O_{1}\right\}\right\} \cup i d, p_{q+1}\left(\tilde{V}^{\prime},\left\langle A_{1}, I\right\rangle, M^{\prime}\right)
\end{array}\right\}
\end{array}\right.
\end{gathered}
$$

Figure 6. Compilation of memory-related instructions.

invoke $S, \kappa \cdot m\left(t_{1}, \ldots, t_{p}\right) t$ where $t \neq$ void. As for Java bytecode, real Dalvik bytecode must pass a verification check before being run on a device. Program points of $P$ are denoted by $q, q^{\prime}, \ldots$ and we let $q+1$ denote the program point immediately following $q$.

Our rules for compiling the instructions of $P$ into clauses are given in Fig. 4-6. Some of them have already been presented in (Payet and Mesnard 2014). They have the form $q$ : ins $\mapsto$ $E$ where $E$ is the set of clauses resulting from the compilation of instruction ins occurring at $q$. We sometimes write $\frac{\psi}{q: i n s \mapsto E}$ meaning that the rule only applies when condition $\psi$ holds.

We assign a predicate symbol $p_{q}$ to each program point $q$ of $P$. The arity of $p_{q}$ is $r+2$ where $r=\operatorname{reg}(\omega)$ and $\omega$ is the method where $q$ occurs. We assign the following meaning to the parameters of $p_{q}$. In an atom of the form

$$
p_{q}\left(V_{0}, \ldots, V_{r-1}, M, M^{\prime}\right)
$$

the first $r+1$ parameters correspond to the state of the current frame just before executing the instruction at $q: V_{0}, \ldots, V_{r-1}$ are the values of the registers and $M$ is the memory. The last parameter $M^{\prime}$ is the memory upon termination of $\omega$. It is used for handling method calls; it is instantiated in the clauses generated for return and its value is used in the clauses generated for invoke (Fig. 5).

We generate clauses with constraints on integer and array terms. Our constraint theory combines the theory of integers with that of arrays defined in (Bradley et al. 2006). We borrow the following notations from (Bradley et al. 2006): the read $a[i]$ returns the value stored at position $i$ of the array $a$ and the write $a\{i \leftarrow e\}$ is $a$ modified so that position $i$ has value $e$; for multidimensional arrays, $a[i] \cdots[j]$ is abbreviated with $a[i, \ldots, j]$. We model a memory as a pair $\langle a, i\rangle$ where $a$ is an array, called memory content, indexed from 0 upwards and $i$ is the index where the next insertion in $a$ will take place. We do not model garbage collection and assume that the memory is unbounded. Memory locations are indexes into $a$; they start at 1 and 0 corresponds to the null value. We model an object as an array of integers $\left[\kappa, x_{0}, \ldots, x_{n}\right]$, indexed from 0 upwards, where $\kappa$ is the class identifier and $x_{0}, \ldots, x_{n}$ are the current values of the fields. Note that the value $x_{j}$ of a field $j$ is located at index $j+1$. A memory content has the form $\left[x, o_{0}, \ldots, o_{n}\right]$ where $x$, an integer, is the result of the most recent called, non-void, method and $o_{0}, \ldots, o_{n}$ are objects. 
Definition 3. Our CLP domain of computation (values interpreting constraints) is $\mathcal{D}=\mathbb{Z} \cup \mathcal{O} \cup \mathcal{A}$ where $\mathcal{O}$ is the set objects and $\mathcal{A}$ is the set of memory contents.

Each rule of Fig. 4-6 considers an instruction ins occurring at a program point $q$. Uppercase letters denote variables. We let $\tilde{V}=$ $V_{0}, \ldots, V_{r-1}$ and $\tilde{V}^{\prime}=V_{0}^{\prime}, \ldots, V_{r-1}^{\prime}$ be sequences of distinct variables where $r$ is the number of registers used by the method where ins occurs. For each $i \in[0, r-1]$, variable $V_{i}$ (resp. $V_{i}^{\prime}$ ) denotes the value of register $i$ before (resp. after) executing ins. We use variables $M, M^{\prime}, \ldots$ or pairs of variables $\langle A, I\rangle,\left\langle A^{\prime}, I^{\prime}\right\rangle, \ldots$. for denoting the memory. We let $i d$ denote the sequence $\left(V_{0}^{\prime}=\right.$ $\left.V_{0}, \ldots, V_{r-1}^{\prime}=V_{r-1}\right)$ and $i d_{-i}$ (where $i \in[0, r-1]$ ) the sequence $\left(V_{0}^{\prime}=V_{0}, \ldots, V_{i-1}^{\prime}=V_{i-1}, V_{i+1}^{\prime}=V_{i+1} \ldots, V_{r-1}^{\prime}=\right.$ $\left.V_{r-1}\right)$. By $|\tilde{W}|$ we mean the length of sequence $\tilde{W}$. For any method $\omega=\kappa \cdot m\left(t_{1}, \ldots, t_{p}\right) t, q_{\omega}$ is the program point where $\omega$ starts. Moreover, for any subclass $\kappa^{\prime}$ of $\kappa$, we let $\operatorname{lookup}\left(\omega, \kappa^{\prime}\right)$ denote the closest method in the superclass chain of $\kappa^{\prime}$ that has name $m$, expects $p$ formal parameters of type $t_{1}, \ldots, t_{p}$, respectively, and returns a value of type $t$. As $\kappa^{\prime}$ is a subclass of $\kappa$ and $\omega=$ $\kappa \cdot m\left(t_{1}, \ldots, t_{p}\right) t$, it is guaranteed that such a method exists.

Some compilation rules are rather straightforward. For instance, const $d, c$ writes constant $c$ into register $d$, so in Fig. 4 the output register variable $V_{d}^{\prime}$ is set to $c$ while the other register variables remain unchanged (modelled with $i d_{-d}$ ). Rules for move, $a d d$ and goto are similar. The rule for if-lt $i, j, q^{\prime}$ generates two clauses expressing that when the test is true execution jumps to program point $q^{\prime}$ otherwise it jumps to the next instruction i.e., to program point $q+1$.

In Fig. 5 we consider method calls. The rule for instruction invoke $s_{0}, \ldots, s_{p}, \kappa \cdot m\left(t_{1}, \ldots, t_{p}\right) t$ works as follows. We impose that $V_{s_{0}}$ (the receiver of the call) is a non-null location (i.e., $V_{s_{0}}>0$ ). Therefore, if $V_{s_{0}} \leq 0$, the execution of the generated CLP program fails, as the original Dalvik program. Moreover, we statically express the Dalvik dynamic lookup of the method to invoke. The method that will effectively be invoked at runtime is necessarily defined in a subclass of $\kappa$. Hence, for each subclass $\kappa^{\prime}$ of $\kappa$, we generate a clause in which we specify that if the class of object $A\left[V_{s_{0}}\right]$ is $\kappa^{\prime}$ (i.e., $\kappa^{\prime}=A\left[V_{s_{0}}, 0\right]$ ), then we invoke the closest method $\omega^{\prime}$ in the superclass chain of $\kappa^{\prime}$ that has name $m$, expects $p$ formal parameters of type $t_{1}, \ldots, t_{p}$ and returns a value of type $t$. This invocation is modelled by a call to $p_{q_{\omega^{\prime}}}$ with a set of registers initialised as needed i.e., the arguments of the call in the last registers and the other registers set to 0 . This call potentially modifies the memory, which yields the new memory $M_{1}$. Finally, when the execution of the invoked method terminates, we transfer control to the program point following the invocation point. This is modelled by a call to $p_{q+1}$ with input memory $M_{1}$. The rule for move-result produces a clause that writes the value returned by the most recent called method (i.e., $A[0]$ ) into register $d$. Instructions return and return $s$ stop the execution of the current method, hence the body of the generated clauses contains no call. Moreover, the clause generated for return $s$ writes the value of register $s$ at index 0 of the memory content i.e., it sets the value returned by the most recent called method to that of register $s$.

Example 4. Let us consider the program in Fig. 7. In method $\mathrm{f}$, the variable a has type $\mathrm{A}$, hence it may store a reference to an instance of any subclass of A. Therefore, when compiling the call a.mm() to clauses, we have to consider all the subclasses of A i.e., A itself and B. For each subclass, we determine the method that would be invoked if a reference to an instance of it was stored in a. If a stores a reference to an instance of $\mathrm{A}$, then the method $\mathrm{mm}$ of $\mathrm{A}$ is invoked. If a stores a reference to an instance of $\mathrm{B}$, then the method $\mathrm{mm}$ of $\mathrm{B}$ is invoked. So, the instruction at line 14 is compiled to:

$$
\begin{gathered}
p_{14}\left(V_{0}, V_{1}, V_{2}, V_{3},\langle A, I\rangle, M^{\prime}\right) \leftarrow\left\{V_{0}>0, \mathrm{~A}=A\left[V_{0}, 0\right],\right. \\
\left.V_{0}^{\prime}=V_{0}, V_{1}^{\prime}=V_{1}, V_{2}^{\prime}=V_{2}, V_{3}^{\prime}=V_{3}\right\}, \\
p_{4}\left(0, V_{0},\langle A, I\rangle, M_{1}\right), p_{15}\left(V_{0}^{\prime}, V_{1}^{\prime}, V_{2}^{\prime}, V_{3}^{\prime}, M_{1}, M^{\prime}\right) . \\
p_{14}\left(V_{0}, V_{1}, V_{2}, V_{3},\langle A, I\rangle, M^{\prime}\right) \leftarrow\left\{V_{0}>0, \mathrm{~B}=A\left[V_{0}, 0\right],\right. \\
\left.V_{0}^{\prime}=V_{0}, V_{1}^{\prime}=V_{1}, V_{2}^{\prime}=V_{2}, V_{3}^{\prime}=V_{3}\right\}, \\
p_{7}\left(0, V_{0},\langle A, I\rangle, M_{1}\right), p_{15}\left(V_{0}^{\prime}, V_{1}^{\prime}, V_{2}^{\prime}, V_{3}^{\prime}, M_{1}, M^{\prime}\right) .
\end{gathered}
$$

Now, consider the call a.m(). As there is no implementation of $\mathrm{m}$ in $\mathrm{B}$, the method $\mathrm{m}$ of $\mathrm{A}$ is always invoked here. So, the instruction at line 13 is compiled to:

$$
\begin{gathered}
p_{13}\left(V_{0}, V_{1}, V_{2}, V_{3},\langle A, I\rangle, M^{\prime}\right) \leftarrow\left\{V_{0}>0, \mathrm{~A}=A\left[V_{0}, 0\right],\right. \\
\left.V_{0}^{\prime}=V_{0}, V_{1}^{\prime}=V_{1}, V_{2}^{\prime}=V_{2}, V_{3}^{\prime}=V_{3}\right\}, \\
p_{0}\left(0, V_{0},\langle A, I\rangle, M_{1}\right), p_{14}\left(V_{0}^{\prime}, V_{1}^{\prime}, V_{2}^{\prime}, V_{3}^{\prime}, M_{1}, M^{\prime}\right) . \\
p_{13}\left(V_{0}, V_{1}, V_{2}, V_{3},\langle A, I\rangle, M^{\prime}\right) \leftarrow\left\{V_{0}>0, \mathrm{~B}=A\left[V_{0}, 0\right],\right. \\
\left.V_{0}^{\prime}=V_{0}, V_{1}^{\prime}=V_{1}, V_{2}^{\prime}=V_{2}, V_{3}^{\prime}=V_{3}\right\}, \\
p_{0}\left(0, V_{0},\langle A, I\rangle, M_{1}\right), p_{14}\left(V_{0}^{\prime}, V_{1}^{\prime}, V_{2}^{\prime}, V_{3}^{\prime}, M_{1}, M^{\prime}\right) .
\end{gathered}
$$

Finally, in Fig. 6 we consider the memory-related instructions. The rule for new-instance $d, \kappa$ builds a new object $O$ which is properly initialised, stores $O$ in memory at location $I$ (i.e., $A_{1}=$ $A\{I \leftarrow O\}$ ), writes the location of $O$ into register $d$ (i.e., $V_{d}^{\prime}=I$ ) and sets the next insertion index to $I+1$ (i.e., $I_{1}=I+1$ ). In the clauses generated for iget $d, i, j$ and iput $s, i, j$ we specify that the value of register $i$ is a non-null location (i.e., $V_{i}>0$ ). Therefore, if $V_{i} \leq 0$, the execution of the generated CLP program fails, as the original Dalvik program. The rule for iget considers the object whose location is in register $i$ (i.e., $A\left[V_{i}\right]$ ) and writes the value of field $j$ of this object into register $d$ i.e., $V_{d}^{\prime}=A\left[V_{i}, j+1\right]$. We write $A\left[V_{i}, j+1\right]$ for accessing field $j$ because the array $A\left[V_{i}\right]$ starts with the class identifier of the object, hence field $j$ is located at index $j+1$. The rule for iput considers the object $O$ whose location is in register $i$ (i.e., $O=A\left[V_{i}\right]$ ) and replaces it in memory with a new object $O_{1}$ (i.e., $A_{1}=A\left\{V_{i} \leftarrow O_{1}\right\}$ ) which is the same as $O$ up to the value of field $j$, which is set from the value of register $s$ (i.e., $O_{1}=O\left\{j+1 \leftarrow V_{s}\right\}$ ).

Example 5. In Fig. 7, objects of class A only have one field (n). Hence, the new-instance instruction at line 11 of the Dalvik program is compiled into the clause:

$$
\begin{gathered}
p_{11}\left(V_{0}, V_{1}, V_{2}, V_{3},\langle A, I\rangle, M^{\prime}\right) \leftarrow\{O[0]=\mathrm{A}, O[1]=0 \\
A_{1}=A\{I \leftarrow O\}, V_{0}^{\prime}=I, I_{1}=I+1, \\
\left.V_{1}^{\prime}=V_{1}, V_{2}^{\prime}=V_{2}, V_{3}^{\prime}=V_{3}\right\} \\
p_{12}\left(V_{0}^{\prime}, V_{1}^{\prime}, V_{2}^{\prime}, V_{3}^{\prime},\left\langle A_{1}, I_{1}\right\rangle, M^{\prime}\right) .
\end{gathered}
$$

\section{Algorithm Recognition in Horn Clauses}

In this section we will define what it means for two (decompiled) programs to be algorithmically equivalent. Proving algorithmic equivalence boils down (Metzger and Wen 2000) to proving semantic equivalence on the one hand (the two program fragments compute the same results and/or exhibit the same behaviour) and some sort of structural equivalence on the other hand, meaning that the two program fragments are similar in their (algorithmic) structure. We will first define what semantic equivalence means in our setting, and we will focus on structural equivalence afterwards.

\subsection{Semantic equivalence of code fragments}

A decompiled Dalvik program is represented by a set of predicate definitions of the form $H \leftarrow\{C\}, \bar{B}$ where $H$ is the head atom, $C$ is a set of constraints and $\bar{B}$ a conjunction of atoms (most notably 
such that $|R|=\left|R^{\prime}\right|=n$. We say that $\left(p_{s}, R\right)$ computes in $P_{1}$ a subset of $\left(p_{q}, R^{\prime}\right)$ in $P_{2}$ if and only if for each call of the form $p_{s}\left(V_{0}, \ldots, V_{n_{s}-3}, M, M^{\prime}\right) \theta$ with computed answer substitution $\theta^{\prime}$, there also exists a call $p_{q}\left(V_{0}, \ldots, V_{n_{q}-3}, M, M^{\prime}\right) \sigma$ with computed answer substitution $\sigma^{\prime}$ such that the following holds for all $k \in 0 \ldots n-1$ :

$$
\begin{aligned}
& \text { 1. }\left(\theta\left(V_{R_{k}}\right), \mu_{\text {in }}\right) \approx\left(\sigma\left(V_{R_{k}^{\prime}}\right), \mu_{\text {in }}^{\prime}\right) \\
& \text { 2. }\left(\theta\left(V_{R_{k}}\right), \mu_{\text {out }}\right) \approx\left(\sigma\left(V_{R_{k}^{\prime}}\right), \mu_{\text {out }}^{\prime}\right) \\
& \text { 3. }\left(a[0], \mu_{\text {out }}\right) \approx\left(a^{\prime}[0], \mu_{\text {out }}^{\prime}\right)
\end{aligned}
$$

where $\mu_{i n}=\theta(M), \mu_{i n}^{\prime}=\sigma(M), \mu_{\text {out }}=\theta^{\prime}\left(M^{\prime}\right)=\langle a, i\rangle$, and $\mu_{\text {out }}^{\prime}=\sigma^{\prime}\left(M^{\prime}\right)=\left\langle a^{\prime}, i^{\prime}\right\rangle$. Moreover, we say that $\left(p_{s}, R\right)$ computes the same in $P_{1}$ as does $\left(p_{q}, R^{\prime}\right)$ in $P_{2}$ if and only if $\left(p_{s}, R\right)$ computes a subset of $\left(p_{q}, R^{\prime}\right)$ and vice versa in their respective programs.

The above definition allows us to characterise predicates as computing the same results, even if these predicates only partially exhibit the same behaviour. Indeed, what matters is that they compute the same return value and update those parts of the memory pointed to by arguments in $R$, respectively $R^{\prime}$, in the same way. The parts of the memory that are pointed to by arguments not comprised in either $R$ or $R^{\prime}$ may be updated differently. Note that at the CLP level, a predicate such as $p_{s}$ or $p_{q}$ will always be called with all but the last argument (representing the output memory) a ground value. Consequently, the computed answer for the call contains a single binding, binding the output memory argument to a ground term. That explains why in condition 2 of the definition we compare $\theta\left(V_{R_{k}}\right)$ and $\sigma\left(V_{R_{k}^{\prime}}\right)$ (the argument values at the time of the call) with respect to the output memories (representing the memory states at the time of the return of the call).

Example 8. Figures 8 and 9 represent two Java methods for computing $x^{n}$ (where $x$ and $n$ are arguments of the method) and the CLP code that was generated from the corresponding Dalvik code (not displayed). In order to make the CLP code more readable, all intermediate non-recursive predicates have been unfolded. ${ }^{1}$ If we call the CLP program of Figure $8 P_{\exp 1}$ and that of Figure 9 $P_{\exp 2}$, it is not hard to see that $\left(p_{1 \_0},\langle 5,4\rangle\right)$ computes the same result in $P_{\text {exp } 1}$ as does $\left(p_{2 \_0},\langle 6,5\rangle\right)$ in $P_{\text {exp } 2 \text {. Indeed, a closer look }}$ at the code makes it clear that the arguments $V_{4}$ and $V_{3}$ in $p_{1 \_}$ represent, respectively, the arguments $n$ and $x$ from the original method whereas in $p_{2 \_}$this role is played by the arguments $V_{5}$ and $V_{4}$. Moreover, from the original Java code it can be clearly seen that both methods compute the same return value for all possible values of the arguments.

The following proposition is trivial to prove:

Proposition 9. The "computes the same" relation defined in Definition 7 is an equivalence relation.

As Example 8 illustrates, it is essential that we consider a subset of arguments when comparing predicates since some of the arguments, introduced by the compilation, are not (or, due to the CLP transformations, no longer) used. This is the case for $V_{2}$ in $p_{1 \_0}$ and both $V_{2}$ and $V_{3}$ in $p_{2 \_}$. Moreover, selecting a subset of arguments allows us to focus on a (sub)computation of interest when considering semantic equivalence of predicates. As a technical note, the programs $P_{e x p 1}$ and $P_{e x p 2}$ both use two helper arguments representing the auxiliary variables $k$ and $w$ (represented in both $p_{1 \_0}$ and $p_{2 \_0}$ by the arguments $V_{0}$ and $V_{1}$ ); therefore we have also that they both compute the same result with respect to an extended set

\footnotetext{
${ }^{1}$ Unfolding is one of the main transformations that we will consider in our transformation-based approach towards structural equivalence, see Section 3.2 .
}

of arguments, notably we have that $\left(p_{1 \_0},\langle 1,2,5,4\rangle\right)$ computes the same as $\left(p_{2_{-} 0},\langle 1,2,6,5\rangle\right)$.

\subsection{Structural equivalence}

As is common practice in the literature on algorithm recognition (see e.g. (Metzger and Wen 2000)), we will define algorithmic equivalence using the notion of program transformation, the basic and intuitive idea being that two programs are algorithmically equivalent if one can be transformed into the other by a series of (semantic-preserving) transformations. However, the fact that we use CLP as the representation language for the algorithms allows us to restrict our attention to a limited number of nonetheless powerful transformations (such as slicing and unfolding) whereas more traditional approaches (Metzger and Wen 2000) usually consider a wide variety of more low-level transformations as they are working on the program's source code (such as renaming variables, loop unrolling, array manipulations, etc.)

Let us consider a given set $\mathcal{R}$ of available program transformations. We will in a moment provide examples of concrete transformations that might be considered in this set but define first the notion of an $\mathcal{R}$-transformation sequence as follows, based on (Pettorossi and Proietti 1998).

Definition 10. Let $\mathcal{R}$ be a set of program transformations and $P$ a CLP program. Then an $\mathcal{R}$-transformation sequence of $P$ is a finite sequence of CLP programs, denoted $\left\langle P_{0}, P_{1}, \ldots, P_{n}\right\rangle$, where $P_{0}=P$ and $\forall i(0<i \leq n): P_{i}$ is obtained by the application of one transformation from $\mathcal{R}$ on $P_{i-1}$.

Given a predefined set of program transformations $\mathcal{R}$ and CLP programs $P$ and $Q$, we will often use $P \rightsquigarrow_{\mathcal{R}}^{*} Q$ to represent the fact that there exists an $\mathcal{R}$-transformation sequence $\left\langle P_{0}, P_{1}, \ldots, P_{n}\right\rangle$ with $P_{0}=P$ and $P_{n}=Q$. Two transformations of particular interest for our purpose are the unfolding (Pettorossi and Proietti 1998) and slicing (Szilágyi et al. 2002) transformations.

Definition 11. Given a program $P$, let c be a clause $A \leftarrow\{C\}, \bar{B}$ in $P, B_{s}$ one of the atoms in $\bar{B}$, and

$$
\left\{\begin{array}{l}
H_{1} \leftarrow\left\{C_{1}\right\}, \bar{L}_{1} \\
\vdots \\
H_{n} \leftarrow\left\{C_{n}\right\}, \bar{L}_{n}
\end{array}\right.
$$

the (renamed apart) set of clauses in $P$ such that $C \wedge C_{i} \wedge\left(B_{s}=\right.$ $\left.H_{i}\right)$ is satisfiable for all $1 \leq i \leq n$. Then unfolding the atom $B_{s}$ in the clause c consists in replacing $c$ by the set of clauses

$$
\left.\left\{A \leftarrow\left\{C \wedge C_{i} \wedge B_{s}=H_{i}\right)\right\},{\overline{B^{\prime}}}_{i} \mid 1 \leq i \leq n\right\}
$$

where $\overline{B^{\prime}}{ }_{i}$ represents the conjunction obtained by replacing, in $\bar{B}$, the atom $B_{s}$ by the conjunction $\bar{L}_{i}$.

Example 12. Reconsider the program $P_{\text {exp } 1}$ defined at the right hand side in Figure 8. Unfolding the single body atom in the clause defining $p_{1 \_0}$ results in the program defined in part (a) of Figure 11.

Definition 13. Given the definition of a predicate $p$ in a program $P$. A slice of $p$ is a predicate $p^{\prime}$ that is obtained from $p$ by removing a (possibly empty) subset of its clauses and removing, for each remaining clause, a (possibly empty) subset of the arguments, constraints and atoms therein.

Example 14. Consider the program $P_{\exp 2}$ depicted on the righthand side of Figure 9. We can compute the slice given in Figure 10 by removing the third and fourth arguments $\left(V_{2}\right.$ and $\left.V_{3}\right)$ from each clause and from each call therein, as well as removing the superfluous atom $V_{2}^{\prime}=1$ from the last clause's body.

While unfolding preserves the computed answer semantics of a program (Pettorossi and Proietti 1998), slicing obviously does not. 
and then the functor +1 is introduced around the first argument of $p_{1 \_2}$ both in the head of the predicate as in each call (part (c)). Note that, in contrast with the slice we computed for $P_{\text {exp } 2}$, only a single argument is removed during the transformation of $P_{\exp 1 .}$. Subsequently, constraint simplification is applied to obtain the final result of the transformation, $P_{\exp }$ (Figure 12). Since each transformation (except the slicing operation) preserves the semantics of the predicate of interest, $p_{1 \_0}$, we trivially have that the transformation sequence $P_{\exp 1} \rightsquigarrow{ }_{\mathcal{R}}^{*} P_{\text {exp }}$ correctly transforms $\left(p_{1 \_0},\langle 4,5\rangle\right)$ into $\left(p_{1 \_},\langle 3,4\rangle\right)$. Note the shift in argument positions due to the argument that was removed in the process. Now, it is not hard to see that the slice we computed of $P_{\exp 2}$ (see Figure 10) is nothing but a renaming of $P_{\exp }$ and we have thus a transformation sequence $P_{\exp 2} \rightsquigarrow_{\mathcal{R}}^{*} P_{\exp }$ that correctly transforms $\left(p_{2_{-} 0},\langle 5,6\rangle\right)$ into $\left(p_{1 \_0},\langle 3,4\rangle\right)$.

The following easy to prove result justifies our definition for algorithmic equivalence by establishing a formal link between structural equivalence as defined by a transformation sequence and semantic equivalence of the involved programs.

Proposition 19. Given programs $P_{1}$ and $P_{2}$, predicates $p_{1}$ and $p_{2}$ and sequences of argument positions $R_{1}$ and $R_{2}$. If $P_{1}$ and $P_{2}$ are algorithmically equivalent for $\left(p_{1}, R_{1}\right)$ and $\left(p_{2}, R_{2}\right)$, then $\left(p_{1}, R_{1}\right)$ computes the same result in $P_{1}$ as does $\left(p_{2}, R_{2}\right)$ in $P_{2}$.

Proof. By algorithmic equivalence (Definition 17) there exist a program $Q$, predicate $p$ and sequence of argument positions $R$ such that $P_{1} \rightsquigarrow{ }_{\mathcal{R}}^{*} Q$ correctly transforms $\left(p_{1}, R_{1}\right)$ and $P_{2} \rightsquigarrow_{\mathcal{R}}^{*} Q$ correctly transforms $\left(p_{2}, R_{2}\right)$. By Definition 16 , it follows that $\left(p_{1}, R_{1}\right)$ computes in $P_{1}$ the same as does $(q, R)$ in $Q$ and, by the same definition and the symmetry of the "computes the same" relation $(q, R)$ computes in $Q$ the same as does $\left(p_{2}, R_{2}\right)$ in $P_{2}$. Transitivity of the "computes the same" relation allows us to conclude the proof.

We conclude this section with a discussion of our approach. Algorithmic equivalence can be seen as an approximation of semantic equivalence, but quite stronger as it incorporates a syntactical component: indeed, the considered algorithms should not only compute the same results, their syntactical representation (at the Horn clause level) should be related by means of the transformations in $\mathcal{R}$. The fact that our notion of algorithmic equivalence is parametrized with this set $\mathcal{R}$ is coherent with the fact that there is no single universally accepted definition for algorithmic equivalence (Blass et al. 2009) and essentially allows us to define a whole hierarchy of characterizations of $\mathcal{R}$-algorithmic equivalence, for different instantiations of $\mathcal{R}$. For instance, by instantiating $\mathcal{R}$ to just $\{i d\}-$ with $i d$ being the identity transformation - we obtain a very strong characterization in which algorithmic equivalence is basically a synonym for having an identical Horn clause representation. Adding more transformations to $\mathcal{R}$ allows for more liberal characterizations of algorithmic equivalence. For instance, when $\mathcal{R}$ is instantiated with the unfolding rule, we obtain an $\mathcal{R}$-algorithmic equivalent criterion that characterises algorithms as equivalent if their Horn clause representation is identical modulo unfolding. Even with a limited set of transformations in $\mathcal{R}$, the definition allows for multiple degrees of liberty when considering algorithmic equivalence. In particular when a slicing transformation is present, algorithms could - in an extreme case - be characterised as equivalent even if they do not share any computation (i.e. when all computations are sliced away in the transformation sequence). This illustrates that the definition, even with a suitable incarnation for $\mathcal{R}$ must be tuned for the application at hand, in particular when the application of interest is related to plagiarism detection.

\section{Related Work}

The seminal idea of translating imperative programs into CLP for static analysis has been introduced in (Peralta et al. 1998), where a semantics-based interpreter of an imperative programming language is expressed as a CLP program. This interpreter together with a term representation of the imperative program to be analyzed is partially evaluated. The residual CLP program is statically analyzed and the results - invariants expressed as linear inequalities between program variables (Cousot and Halbwachs 1978) - are brought back to the initial imperative program. As another example, (De Angelis et al. 2015) proposes a method for automatically generating verification conditions for imperative programs by program specialization. The approach of (Peralta et al. 1998) has also been applied to Object-Oriented programs. For instance, the Java bytecode static analyzer Julia (Spoto et al. 2010) is able to generate a CLP program whose termination implies the termination of the initial Java bytecode program. As a last example, in (Albert et al. 2012) Java bytecode programs are rewritten into a rule based formalism similar to Horn clauses. Then, given a cost model, cost relations are derived. A cost analysis is automatically inferred by solving such cost relations with the help of a dedicated constraint solver.

Algorithm recognition is a well-established topic in program analysis. We present below some of the main existing works.

Two of the oldest related research projects are the MIT's Programmer's Apprentice (see e.g., (Rich et al. 1979)) and the Knowledge Based Software Assistant, a research program funded by the United States Air Force (see e.g., (Green et al. 1983)). The underlying idea was to adapt artificial intelligence techniques to help software development.

As an offspring of the Programmer's Apprentice, Linda Mills describes an automated program recognition system in (Wills 1990, 1993). It aims at helping software maintenance, translation, and debugging. Given a program and a library of clichés, i.e., programming stereotypes and associated structures, the system builds a hierarchical description of the program in terms of the clichés found and their relationships.

In (Martino and Iannello 1996), the authors present a tool called PAP Recognizer, where PAP stands for Parallelizable Algorithmic Patterns. It implements a plan-based technique for the hierarchical recognition of concept-instances in the program. It aims at automatically parallelizing the code. Another approach to automatically replace the sequential parts of a program with their parallelized versions is described in (Metzger and Wen 2000). Although similar to the previous work, this approach focusses on the computationnally intensive parts of the program.

While most techniques for algorithm recognition are based on using some kind of pattern or template matching, others try to capture the essence of the algorithm at hand. In (Alias and Barthou 2003), for example, algorithms are converted into a system of recurrence equations. In (Taherkhani 2011; Taherkhani and Malmi 2013), Ahmad Taherkhani proposes to statically summarize programs by means of software metrics and program schemas. Then a decision tree classifier acts as an algorithm recognizer. The approach is evaluated by classifying sorting algorithms written by students.

More recently, (Zhang et al. 2012) specifically targets algorithm plagiarism detection. Their detection mechanism is based on abstracting the algorithm by a signature that is computed from a sequence of core values that should arise in any implementation of the algorithm. One of the advantages of such value based signature is the resilience with respect to several obfuscation techniques. 


$$
\begin{aligned}
p_{1 \_0} & \left(V_{0}, V_{1}, V_{2}, V_{3}, V_{4},\langle A, I\rangle,\left\langle A^{\prime}, I\right\rangle^{\prime}\right) \leftarrow \\
& \left\{V_{4} \leq 0, A^{\prime}=A\{0 \leftarrow 1\}\right\} . \\
p_{1 \_0} & \left(V_{0}, V_{1}, V_{2}, V_{3}, V_{4}, M, M^{\prime}\right) \leftarrow \\
& \left\{V_{4}>0, V_{0}^{\prime}=V_{4}-1, V_{1}^{\prime}=1 * V_{3}\right\}, \\
& p_{1 \_2}\left(V_{0}^{\prime}, V_{1}^{\prime}, V_{2}, V_{3}, V_{4}, M, M^{\prime}\right) . \\
p_{1 \_2} & \left(V_{0}, V_{1}, V_{2}, V_{3}, V_{4},\langle A, I\rangle,\left\langle A^{\prime}, I\right\rangle\right) \leftarrow \\
& \left\{V_{0} \leq 0, A^{\prime}=A\left\{0 \leftarrow V_{1}\right\}\right\} . \\
p_{1 \_2} & \left(V_{0}, V_{1}, V_{2}, V_{3}, V_{4}, M, M^{\prime}\right) \leftarrow \\
& \left\{V_{0}>0, V_{0}^{\prime}=V_{0}-1, V_{1}^{\prime}=V_{1} * V_{3}\right\}, \\
& p_{1 \_2}\left(V_{0}^{\prime}, V_{1}^{\prime}, V_{2}, V_{3}, V_{4}, M, M^{\prime}\right) .
\end{aligned}
$$

(a)

$$
\begin{gathered}
p_{1 \_0}\left(V_{0}, V_{1}, V_{3}, V_{4},\langle A, I\rangle,\left\langle A^{\prime}, I\right\rangle^{\prime}\right) \leftarrow \\
\left\{V_{4} \leq 0, A^{\prime}=A\{0 \leftarrow 1\}\right\} \\
p_{1 \_0}\left(V_{0}, V_{1}, V_{3}, V_{4}, M, M^{\prime}\right) \overleftarrow{ } \\
\left\{V_{4}>0, V_{0}^{\prime}=V_{4}-1, V_{1}^{\prime}=1 * V_{3}\right\}, \\
p_{1 \_2}\left(V_{0}^{\prime}, V_{1}^{\prime}, V_{3}, V_{4}, M, M^{\prime}\right) . \\
p_{1 \_2}\left(V_{0}, V_{1}, V_{3}, V_{4},\langle A, I\rangle,\left\langle A^{\prime}, I\right\rangle\right) \leftarrow \\
\left\{V_{0} \leq 0, A^{\prime}=A\left\{0 \leftarrow V_{1}\right\}\right\} \\
p_{1 \_2}\left(V_{0}, V_{1}, V_{3}, V_{4}, M, M^{\prime}\right) \overleftarrow{ } \\
\left\{V_{0}>0, V_{0}^{\prime}=V_{0}-1, V_{1}^{\prime}=V_{1} * V_{3}\right\} \\
\\
p_{1 \_2}\left(V_{0}^{\prime}, V_{1}^{\prime}, V_{3}, V_{4}, M, M^{\prime}\right) .
\end{gathered}
$$

(b)

$$
\begin{aligned}
p_{1 \_0} & \left(V_{0}, V_{1}, V_{3}, V_{4},\langle A, I\rangle,\left\langle A^{\prime}, I\right\rangle^{\prime}\right) \leftarrow \\
& \left\{V_{4} \leq 0, A^{\prime}=A\{0 \leftarrow 1\}\right\} . \\
p_{1 \_0} & \left(V_{0}, V_{1}, V_{3}, V_{4}, M, M^{\prime}\right) \overleftarrow{ } \\
& \left\{V_{4}>0, V_{0}^{\prime}=V_{4}-1, V_{1}^{\prime}=1 * V_{3}\right\}, \\
& p_{1 \_2}\left(V_{0}^{\prime}+1, V_{1}^{\prime}, V_{3}, V_{4}, M, M^{\prime}\right) . \\
p_{1 \_2} & \left(V_{0}+1, V_{1}, V_{3}, V_{4},\langle A, I\rangle,\left\langle A^{\prime}, I\right\rangle\right) \leftarrow \\
& \left\{V_{0} \leq 0, A^{\prime}=A\left\{0 \leftarrow V_{1}\right\}\right\} \\
p_{1 \_2} & \left(V_{0}+1, V_{1}, V_{3}, V_{4}, M, M^{\prime}\right) \leftarrow \\
& \left\{V_{0}>0, V_{0}^{\prime}=V_{0}-1, V_{1}^{\prime}=V_{1} * V_{3}\right\}, \\
& p_{1 \_2}\left(V_{0}^{\prime}+1, V_{1}^{\prime}, V_{3}, V_{4}, M, M^{\prime}\right) .
\end{aligned}
$$

Figure 11. The transformation of program $P_{\text {exp } 1}$ from Example 8 .

(c)

$$
\begin{aligned}
p_{1 \_0} & \left(V_{0}, V_{1}, V_{3}, V_{4},\langle A, I\rangle,\left\langle A^{\prime}, I\right\rangle^{\prime}\right) \leftarrow \\
& \left\{V_{4} \leq 0, A^{\prime}=A\{0 \leftarrow 1\}\right\} . \\
p_{1 \_0} & \left(V_{0}, V_{1}, V_{3}, V_{4}, M, M^{\prime}\right) \leftarrow \\
& \left\{V_{4}>0\right\} \\
& p_{1 \_2}\left(V_{4}, V_{3}, V_{3}, V_{4}, M, M^{\prime}\right) . \\
p_{1 \_2} & \left(V_{0}, V_{1}, V_{3}, V_{4},\langle A, I\rangle,\left\langle A^{\prime}, I\right\rangle\right) \leftarrow \\
& \left\{V_{0} \leq 1, A^{\prime}=A\left\{0 \leftarrow V_{1}\right\}\right\} . \\
p_{1 \_2} & \left(V_{0}, V_{1}, V_{3}, V_{4}, M, M^{\prime}\right) \leftarrow \\
& \left\{V_{0}>1, V_{0}^{\prime}=V_{0}-1, V_{1}^{\prime}=V_{1} * V_{3}\right\}, \\
& p_{1 \_2}\left(V_{0}^{\prime}, V_{1}^{\prime}, V_{3}, V_{4}, M, M^{\prime}\right) .
\end{aligned}
$$

Figure 12. The transformed program $P_{e x p}$.

\section{Conclusion}

We have presented a generic approach to algorithm recognition in binary code. Its genericity stems from two points. On the one hand, the technique is generic with respect to the input language, as soon as we can translate it into an Horn-clause based representation mimicking the operational semantics of the original target processor. On the other hand, the approach is generic with respect to the notion of algorithmic equivalence, via its parametric set of program transformation rules.

One key aspect of our approach is the use of Horn clauses as a language for representing what is basically the model of the algorithms being compared. In addition to the before-mentioned genericity advantage, the use of Horn clauses allows one to instantiate $\mathcal{R}$ using a limited number of powerful, general, and wellunderstood transformations such as unfolding, folding and slicing without the need to resort to more low-level and less-general (or language-dependent) transformations. Nevertheless, the question remains about what are desirable incarnations of $\mathcal{R}$ and whether one should impose restrictions on the transformation sequences used in the proof of algorithmic equivalence.

While a general equivalence relation on algorithms might not exist (Blass et al. 2009), suitable incarnations of $\mathcal{R}$ will most probably depend on the particular application at hand. Defining such an incarnation (and the particular notion of algorithmic equivalence that comes with it) remains an open and challenging question, in particular when applications such as plagiarism detection are concerned.

Future work will focus on developing other front-ends dealing with various input languages as well as on investigating the feasibility of implementing the procedure described in Section 3 for a limited instantiation of $\mathcal{R}$. Even when only a limited number of transformations are present in $\mathcal{R}$, developing a search procedure trying to construct an $\mathcal{R}$-transformation sequence is a non-trivial and daunting task that might need guidance and global analysis of the program's at hand.

\section{References}

E. Albert, P. Arenas, S. Genaim, G. Puebla, and D. Zanardini. Cost analysis of object-oriented bytecode programs. Theor. Comput. Sci., 413(1):142$159,2012$.

C. Alias and D. Barthou. Algorithm recognition based on demand-driven data-flow analysis. In Proceedings of the 10th Working Conference on Reverse Engineering (WCRE), pages 296-305, 2003.

Android developers. http://developer.android.com.

ART and Dalvik. http://source. android.com/devices/tech/dalvik/.

A. Blass, N. Dershowitz, and Y. Gurevich. When are two algorithms the same? Bull. Symbolic Logic, 15(2):145-168, 062009.

A. R. Bradley, Z. Manna, and H. B. Sipma. What's decidable about arrays? In E. A. Emerson and K. S. Namjoshi, editors, Proc. of VMCAI'06, volume 3855 of $L N C S$, pages 427-442. Springer, 2006.

P. Cousot and N. Halbwachs. Automatic discovery of linear restraints among variables of a program. In Conference Record of the Fifth Annual ACM Symposium on Principles of Programming Languages, POPL'78, Tucson, Arizona, USA, January 1978, pages 84-96, 1978.

C. Dandois and W. Vanhoof. Semantic code clones in logic programs. In E. Albert, editor, Proc. of the 22nd International Symposium on LogicBased Program Synthesis and Transformation (LOPSTR'12), volume 7844 of LNCS, pages 35-50. Springer, 2012.

E. De Angelis, F. Fioravanti, A. Pettorossi, and M. Proietti. Semanticsbased generation of verification conditions by program specialization. In Proceedings of the 17th International Symposium on Principles and Practice of Declarative Programming, Siena, Italy, July 14-16, 2015, pages 91-102, 2015.

G. Gange, J. A. Navas, P. Schachte, H. Søndergaard, and P. J. Stuckey. Horn clauses as an intermediate representation for program analysis and transformation. TPLP, 15(4-5):526-542, 2015.

C. Green, D. Luckham, R. Balzer, T. Cheatham, and C. Rich. Report on a knowledge-based software assistant. Technical report, Kestrel Institute, 1983.

J. Jaffar and J. L. Lassez. Constraint logic programming. In Proc. of the ACM Symposium on Principles of Programming Languages, pages 111119. ACM, 1987.

J. Jaffar, M. J. Maher, K. Marriott, and P. J. Stuckey. The semantics of constraint logic programs. Journal of Logic Programming, 37(1-3):146, 1998 .

T. Lindholm and F. Yellin. The Java ${ }^{T M}$ Virtual Machine Specification. Addison-Wesley, second edition, 1999.

J. W. Lloyd. Foundations of Logic Programming. Springer-Verlag, 1987.

B. D. Martino and G. Iannello. PAP recognizer: A tool for automatic recognition of parallelizable patterns. In 4th International Workshop on Program Comprehension (WPC), page 164, 1996. 
R. Metzger and Z. Wen. Automatic Algorithm Recognition and Replacement. The MIT Press, 2000.

E. Payet and F. Mesnard. Non-termination of Dalvik bytecode via compilation to CLP. In C. Fuhs, editor, Proc. of the 14th International Workshop on Termination (WST'14), pages 65-69, 2014.

J. C. Peralta, J. P. Gallagher, and H. Saglam. Analysis of imperative programs through analysis of constraint logic programs. In Static Analysis, 5th International Symposium, SAS '98, Pisa, Italy, September 14-16, 1998, Proceedings, pages 246-261, 1998.

A. Pettorossi and M. Proietti. Transformation of logic programs. In Handbook of Logic in Artificial Intelligence and Logic Programming, volume 5, pages 697-787. Oxford University Press, 1998.

C. Rich, H. E. Shrobe, and R. C. Waters. Overview of the programmer's apprentice. In Proceedings of the Sixth International Joint Conference on Artificial Intelligence (IJCAI), pages 827-828, 1979.

Smali assembler for the dex format. https://github.com/JesusFreke/smali.

F. Spoto, F. Mesnard, and É. Payet. A termination analyzer for Java bytecode based on path-length. ACM Trans. Program. Lang. Syst., 32 (3), 2010.

M. D. Storey. Theories, methods and tools in program comprehension: Past, present and future. In 13th International Workshop on Program Comprehension (IWPC), pages 181-191, 2005.

G. Szilágyi, T. Gyimóthy, and J. Małuszyński. Static and dynamic slicing of constraint logic programs. Automated Software Engineering, 9(1): 41-65, 2002.

A. Taherkhani. Using decision tree classifiers in source code analysis to recognize algorithms: An experiment with sorting algorithms. Comput. J., 54(11):1845-1860, 2011.

A. Taherkhani and L. Malmi. Beacon- and schema-based method for recognizing algorithms from students' source code. Journal of Educational Data Mining, 5(2):69-101, 2013.

L. M. Wills. Automated program recognition: A feasibility demonstration. Artificial Intelligence, 45(1-2):113-171, 1990.

L. M. Wills. Flexible control for program recognition. In Proceedings of Working Conference on Reverse Engineering (WCRE), pages 134-143, 1993.

F. Zhang, Y.-C. Jhi, D. Wu, P. Liu, and S. Zhu. A first step towards algorithm plagiarism detection. In Proceedings of the 2012 International Symposium on Software Testing and Analysis, ISSTA 2012, pages 111121. ACM, 2012.

F. Zhang, H. Huang, S. Zhu, D. Wu, and P. Liu. Viewdroid: Towards obfuscation-resilient mobile application repackaging detection. In Proceedings of the 2014 ACM Conference on Security and Privacy in Wireless and Mobile Networks, WiSec '14, pages 25-36. ACM, 2014. 